\title{
Regrafting of the Split-Thickness Skin Graft Donor-Site: Is It Beneficial?
}

\author{
Catherine M. Legemate, MD ${ }^{*}, \dagger, \oplus$ Ymke Lucas, $M D,{ }^{\dagger}$ Irma M. M. H. Oen, $M D,{ }^{\dagger}$ and \\ Cornelis H. van der Vlies, MD, $\mathrm{PhD}^{\dagger, \ddagger}$
}

\begin{abstract}
Split-thickness skin grafting remains a fundamental treatment for patients with deep burns and other traumatic injuries. Unfortunately, the donor site wound that remains after split skin graft (SSG) harvesting may also cause problems for the patient; they can lead to discomfort and scars with a poor cosmetic outcome. Regrafting of the donor site is one of the methods described to improve donor site healing and scarring. In this report, we describe a case of a 26 -year-old woman with a self-inflicted chemical burn $(0.5 \%$ TBSA $)$ who underwent split skin grafting. During surgery, only part of the donor site was regrafted with split skin graft remnants. This part healed faster and had a better scar quality at 3 months postsurgery. Nevertheless, the appearance and patients' opinion on the regrafted part deteriorated after 12 months. With this case report, we aim to create awareness of the long-term consequences of regrafting, which may differ from short-time results. Patients expected to have poor reepithelialization potential may benefit from regrafting of the SSG on the donor site. But in healthy young individuals, timewise there would be no benefit since it can lead to an aesthetically displeasing result.
\end{abstract}

Split-thickness skin grafting is a common reconstructive technique for the treatment of patients with deep burns and other traumatic injuries. The management of the donor site after harvesting a split-thickness skin graft (SSG) remains up for discussion as many practitioners have varying methods of caring for these wounds, ranging from different types of wound dressings to more innovative techniques. Most studies on donor sites focus on early and rapid reepithelialization but lack data on (long-term) scar appearance, despite the fact that these scars may have a psychological impact and can affect quality of life.

Regrafting of the donor site is one of the methods described to improve donor site healing and their cosmetic outcome. ${ }^{1-4}$ Thompson was the first to describe the value of placing thin skin grafts on donor sites to improve healing. ${ }^{5}$ He noted that donor sites without any grafts had a propensity for hypertrophic scars that were cosmetically unfavorable and that donor sites with thin grafts healed much more quickly with better quality healing. Several other studies described a better aesthetic appearance of donor site scars after regrafting of the donor site. In some of these studies, a larger amount of donor

\footnotetext{
*Department of Plastic, Reconstructive and Hand Surgery, Amsterdam UMC, Vrije Universiteit Amsterdam, Amsterdam Movement Sciences, de Boelelaan 1117, Amsterdam, the Netherlands; ${ }^{+}$Burn Center, Maasstad Hospital, PO Box 9100, 3007 AC Rotterdam, the Netherlands Rotterdam, the Netherlands; ${ }^{\ddagger}$ Department of Surgery, Erasmus $M C$, University Medical Center Rotterdam, Trauma Research Unit, PO Box 2040 Rotterdam, The Netherlands

Conflict of interest statement. None declared.

Funding: None.

Address correspondence to Catherine M. Legemate, MD, Burn Centre, Maasstad Hospital, PO Box 9100, 3007 AC Rotterdam, the Netherlands. Email: legematec@maasstadziekenhuis.nl

(C) American Burn Association 2020. All rights reserved. For permissions, please e-mail: journals.permissions@oup.com.

doi:10.1093/jbcr/ivz166
}

skin was harvested on purpose to cover not only the defect, but also the donor site wound. ${ }^{2,3}$ Others harvested a separate thin SSGs to cover the donor site wound or used SSG remnants to assist donor site healing. ${ }^{1,4}$ However, only one study concerning scar quality used a control group and measured scar quality after 1 year. ${ }^{4}$ The other studies are limited by the lack of an equivalent control group and adequate follow-up period. . $^{2,3}$

We present a case in which only a part of the donor site was regrafted with leftover SSG after coverage of a burn wound. In this patient, scar quality and appearance of the regrafted part were better during the first 3 months, but deteriorated 1 year postsurgery. This case illustrates the importance of a long-term follow-up and a good control group (or intrapatient controlled design) to evaluate final scar appearance and to determine the benefits of techniques that are used to improve donor site outcome.

\section{THE CASE}

A 26-year-old woman was admitted to our division with a self-inflicted chemical burn $(0.5 \%$ TBSA) on her left lower leg, diagnosed as a full-thickness burn. She had a history of nonfatal deliberate self-harm but no other comorbidities. Within 1 week the burn was excised and grafted with a splitthickness autograft, meshed, and expanded 1:1.5. The thickness of the SSG was $0.2 \mathrm{~mm}$ and harvested from her left upper leg. The excess of SSG that was left after coverage of the burn wound was regrafted on the proximal site of the donor site wound (Figure 1). The wound was covered with Kaltostat, an alginate dressing, which was removed after 2 weeks. Reepithelialization (defined as $>95 \%$ ) of the regrafted part was complete within 2 weeks (Figure 2), whereas complete reepithelialization of the nongrafted part took 25 days. The patient told us that part of the wound remained open 


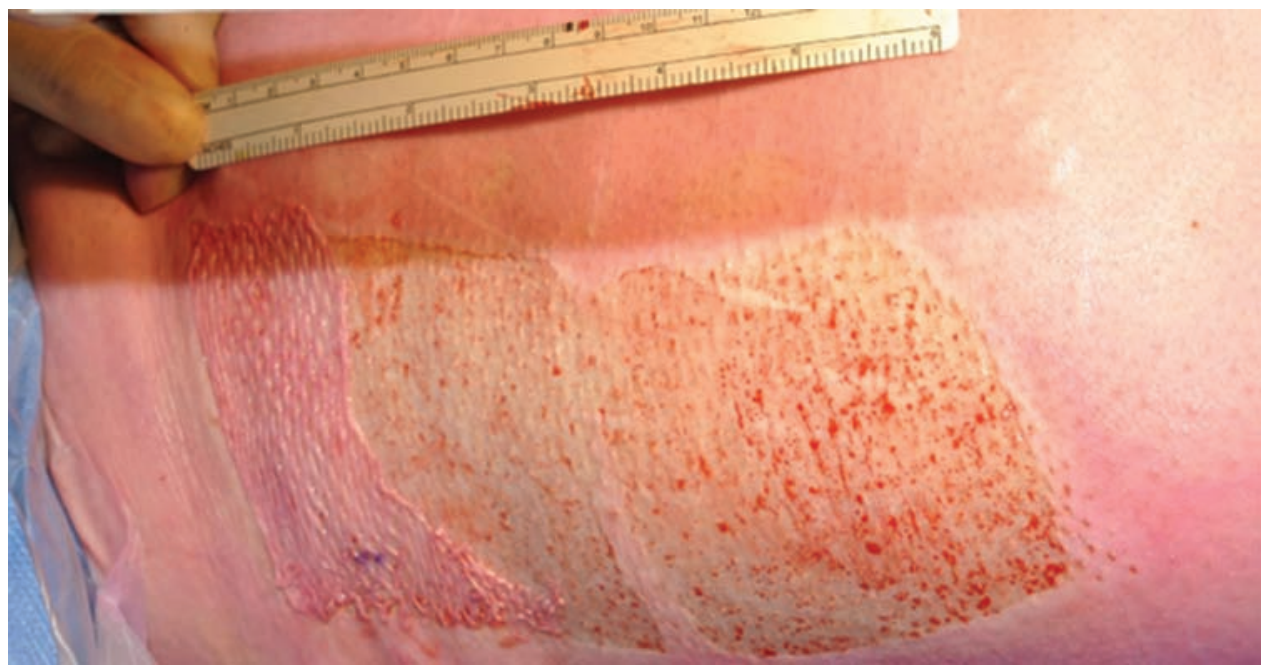

Figure 1. Regrafting of the donor site with leftover SSG on the proximal site of the donor site wound during surgery.

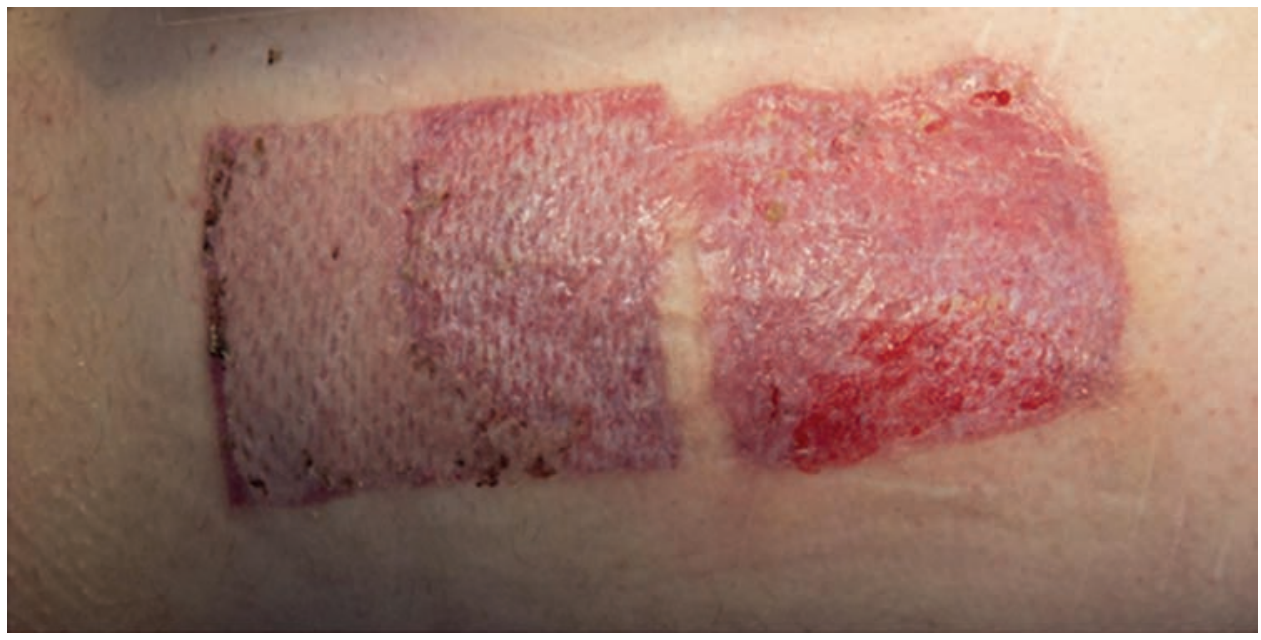

Figure 2. Healing of the donor site wound 3 weeks post-surgery.

due to pruritus and the scratching that she could not control while sleeping (Figure 2). During routine follow-up at 3 months, the regrafted part of the donor site showed less erythema and hypopigmentation (Figure 3 ) and scored better on the overall opinion items of the Patient and Observer Scar Assessment Scale (POSAS) than the nongrafted part (Table 1). Furthermore, the patient expressed her desire for the whole donor site to be regrafted. After 12 months postsurgery, the patient returned to the outpatient clinic for a routine control. Her opinion on the appearance of the two different sites of the donor site scar was altered and turned in favor of the nongrafted part (Table 1). The nongrafted part was only a little erythematous whilst the grafted part was a mix of hypo- and hyperpigmentation. Also, the surface looked and felt more irregular (Figure 4). The overall opinion part of the patient score of the POSAS was 2 for the grafted site and 1 (best scar imaginable) for the nongrafted site.

\section{DISCUSSION}

With the improved survival of patients with burns, scarring became an important focus of clinical burn care. Many studies have described scar quality of the recipient site after split skin grafting in burn patients, whilst studies concerning scar quality of the donor site are limited. In addition, these studies focus mostly on the use of certain wound dressings with wound healing and cost-effectiveness as outcome parameters. $^{6-9}$ Intentional regrafting of the donor site after SSG harvesting is one of the techniques used in daily practice. ${ }^{2-4}$ During these procedures, the surgeon takes a larger amount than necessary or an additional graft, which is than meshed to cover both donor sites. The hypothesis behind this technique is that coverage of the donor site with the SSG may reduce the mechanical force on the wound thereby reducing healing time resulting in fewer complications, less hypertrophic scarring, and improved cosmetic results. ${ }^{5}$ This can be particularly beneficial in patients in which wound healing may be a concern. The drawback of this technique is that the donor site has to be extended with possibly more complications and a larger scar. Therefore, this procedure remains controversial. Regrafting of donor sites might also be done with SSG leftovers, in case the surgeon accidently harvests excessive skin and rather salvages than discards the skin graft remnants. 


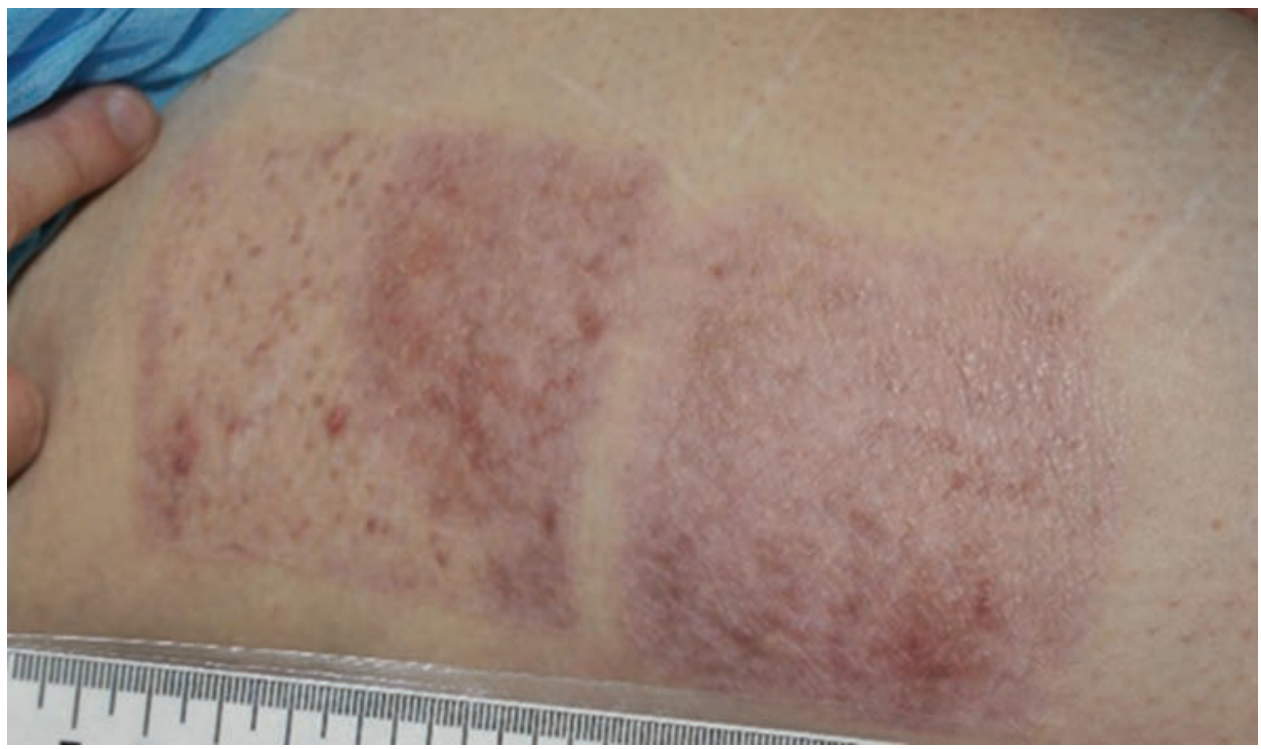

Figure 3. Donor site appearance 3 months post-surgery.

Table 1. POSAS scores* of the regrafted and nongrafted part at 3 and 12 months postsurgery

\begin{tabular}{|c|c|c|c|c|c|c|}
\hline & Regrafted part & Non grafted part & Mean difference & Regrafted part & Non grafted part & Mean difference \\
\hline & 3 months & 3 months & 3 months & 12 months & 12 months & 3 months \\
\hline \multicolumn{7}{|l|}{ Patient scale } \\
\hline Pain & 1 & 1 & 0 & 1 & 1 & 0 \\
\hline Itch & 2 & 5 & 3 & 1 & 1 & 0 \\
\hline Color & 2 & 6 & 4 & 4 & 2 & $2^{\dagger}$ \\
\hline Pliability & 2 & 4 & 2 & 1 & 1 & 0 \\
\hline Thickness & 1 & 1 & 0 & 1 & 1 & 0 \\
\hline Relief & 1 & 1 & 0 & 3 & 1 & $2^{\dagger}$ \\
\hline Mean POSAS & 1.5 & 3 & 1.5 & 1.8 & 1.2 & $0.6^{\dagger}$ \\
\hline Overall Opinion & 2 & 6 & 4 & 2 & 1 & $1^{\dagger}$ \\
\hline \multicolumn{7}{|l|}{ Observer Scale } \\
\hline Vascularity & 3 & 5 & 2 & 1 & 2 & 1 \\
\hline Pigmentation & 2.5 & 5 & 2.5 & 2 & 1 & $1^{\dagger}$ \\
\hline Thickness & 1 & 1 & 0 & 1 & 1 & 0 \\
\hline Relief & 1.5 & 2.5 & 1 & 2 & 1 & $1^{\dagger}$ \\
\hline Pliability & 1 & 2 & 1 & 1 & 1 & 0 \\
\hline Surface Area & 1 & 1 & 0 & 1 & 1 & 0 \\
\hline Mean POSAS & 1.7 & 2.8 & 1.1 & 1.5 & 1.2 & $0.3^{\dagger}$ \\
\hline Overall Opinion & 2.5 & 4.5 & 2 & 2 & 1 & $1^{\dagger}$ \\
\hline
\end{tabular}

* A lower POSAS score correlates with a better scar; a score of 10 reflects the worst imaginable scar.

tDifference in favor of the nongrafted part.

After Thompson et al described better scar quality of donor sites after regrafting, several other studies reported quicker reepithelialization, reduced pain scores, and a good cosmetic appearance without an increase in complications. ${ }^{1-5}$ However, studies with long-term results on donor site outcome are lacking. Bian et al were the only investigators who used a validated scale (the Vancouver Scar Scale) and a control group to assess scar quality and reported a beneficial outcome of regrafting compared with occlusive hydrocellular dressings and paraffin gauze at 1 year follow-up. ${ }^{4}$

Bradow et al stated that it would be difficult to argue against the value of routinely regrafting in the patients expected to have poor reepithelialization potential but that not all patients may benefit of regrafting because of the aesthetically displeasing result that is typical for a meshed skin graft. ${ }^{1}$

Our case shows that in healthy, young individuals the additional value of regrafting is indeed not so clear, since the appearance and patients' opinion of the regrafted site changed over time. Our case is a clear example of how the regrafted part can eventually develop into an aesthetically worse result with the displeasing aspects of a meshed skin graft (e.g., mixed pigmentation, irregular surface) after initial encouraging results, even though the non-grafted part took longer to heal. With this case report, we aim to emphasize the importance of 


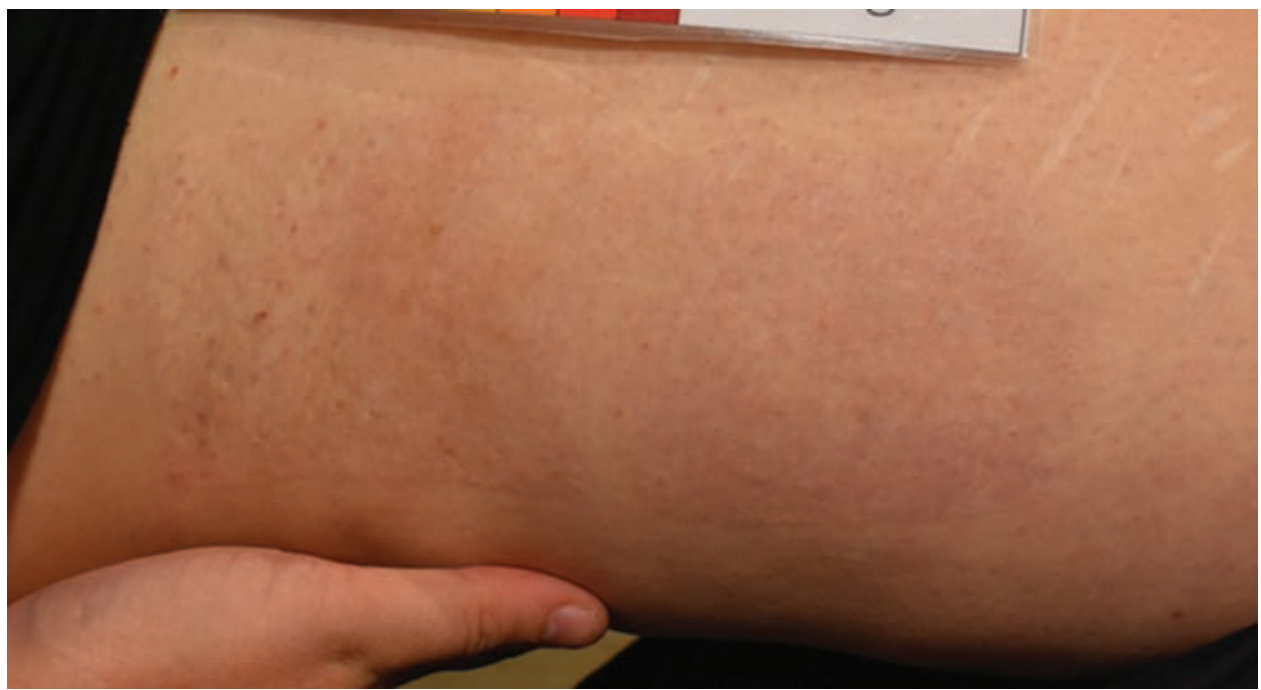

Figure 4. Donor site appearance 12 months post-surgery.

a matched control group (or intrapatient control site) when assessing scar quality in the evaluation of techniques that are used to improve donor site healing. Moreover, follow-up of at least 1 year is needed to draw final conclusions on scar appearance as scars take at least 1 year to mature. ${ }^{10}$

\section{CONCLUSION}

Regrafting of any excess SSG on the donor site is not necessarily best practice in every patient, and the surgeon needs to be aware of the consequences and long-term results. With this case report, we aim to create awareness of the long-term consequences of regrafting, which may differ from short-time results. Patients expected to have poor reepithelialization potential may benefit from regrafting of the SSG on the donor site. But in healthy young individuals, there might be no benefit over time because it could lead to an aesthetically displeasing result.

\section{ACKNOWLEDGMENTS}

Not applicable.

\section{REFERENCES}

1. Bradow BP, Hallock GG, Wilcock SP. Immediate regrafting of the split thickness skin graft donor site assists healing. Plast Reconstr Surg Glob Open 2017;5:e1339.

2. Ablaza VJ, Berlet AC, Manstein ME. An alternative treatment for the split skin-graft donor site. Aesthetic Plast Surg 1997;21:207-09.

3. Goverman J, Kraft CT, Fagan S, Levi B. Back grafting the split-thickness skin graft donor site. J Burn Care Res 2017;38:e443-49.

4. Bian Y, Sun C, Zhang X, et al. Wound-healing improvement by resurfacing split-thickness skin donor sites with thin split-thickness grafting. Burns 2016;42:123-30.

5. Thompson N. A clinical and histological investigation into the fate of epithelial elements buried following the grafting of "shaved" skin surfaces based on a study of the healing of split-skin graft donor sites in man. Br J Plast Surg 1960;13:219-42.

6. Wiechula R. The use of moist wound-healing dressings in the management of split-thickness skin graft donor sites: a systematic review. Int J Nurs Pract 2003;9:S9-17.

7. Voineskos SH, Ayeni OA, McKnight L, Thoma A. Systematic review of skin graft donor-site dressings. Plast Reconstr Surg 2009;124:298-06.

8. Rakel BA, Bermel MA, Abbott LI, et al. Split-thickness skin graft donor site care: a quantitative synthesis of the research. Appl Nurs Res 1998;11:174-82.

9. Canadian Agency for Drugs and Technologies in Health. Dressings and care of skin graft sites: a review of clinical evidence and guidelines [internet]. CADTH rapid response report: summary with critical appraisal. 2013 .

10. van der Wal MB, Vloemans JF, Tuinebreijer WE, et al. Outcome after burns: an observational study on burn scar maturation and predictors for severe scarring. Wound Repair Regen 2012;20:676-87. 